\title{
The effect of anatomic location of injury on mortality risk in a resource-poor setting
}

\author{
Jessica Eaton ${ }^{\mathrm{a}, \mathrm{b}}$, Joanna Grudziak ${ }^{\mathrm{c}}$, Asma Bilal Hanif ${ }^{\mathrm{d}}$, Wanangwa C. Chisenga ${ }^{\mathrm{d}}$, \\ Eldad Hadar ${ }^{\mathrm{e}}$, Anthony Charles ${ }^{\mathrm{a}, \mathrm{c}, \mathrm{d}, *}$
}

${ }^{a}$ UNC -Project Malawi, Lilongwe, Malawi

${ }^{\mathrm{b}}$ University of Louisville School of Medicine, Louisville, KY, United States

${ }^{\mathrm{c}}$ Department of Surgery, University of North Carolina, Chapel Hill, NC, United States

d Department of Surgery, Kamuzu Central Hospital, Lilongwe, Malawi

e Department of Neurosurgery, University of North Carolina, Chapel Hill, NC, United States

A R T I C L E I N F O

Keywords:

Traumatic brain injury

Traumatic spine injury

Head injury

Mortality

Anatomic location of injury

\begin{abstract}
A B S T R A C T
Introduction: Injury is a significant cause of death, with approximately 4.7 million people mortalities each year. By 2030, injury is predicted to be among the top 20 causes of death worldwide. We sought to characterize and compare the mortality probability in trauma patients in a resource-poor setting based on anatomic location of injury.

Methods: We performed a retrospective analysis of prospectively collected data using the trauma database at Kamuzu Central Hospital $(\mathrm{KCH})$ in Lilongwe, Malawi. We included all adult trauma patients ( $\geq$ 16years) admitted between 2011 and 2015. We stratified patients according to anatomic location of injury, and used descriptive statistics to compare characteristics and management of each group. Bivariate analysis by mortality was done to determine covariates for our adjusted model. A Cox proportional hazard model was performed, using upper extremity injury as the baseline comparator. Descriptive statistics were used to describe the trend in incidence and mortality of head and spine injuries over five years.

Results: Of the 76,984 trauma patients who presented to $\mathrm{KCH}$ from 2011 to 2015, 49,126 (63.8\%) were adults, and $8569(17.4 \%)$ were admitted. The most common injury was to the head or spine, seen in 3712 patients (43.6\%). The highest unadjusted hazard ratio for mortality was in head and spine injury patients, at 3.685 (95\% CI=2.50-5.44), which increased to 4.501 (95\% CI =2.78-7.30) when adjusted for age, sex, injury severity, transfer status, injury mechanism, and surgical intervention. Abdominal trauma had the second highest adjusted hazard of mortality, at $3.62(95 \% \mathrm{CI}=1.92-6.84)$ followed by thoracic trauma ( $\mathrm{HR}=1.3621,95 \% \mathrm{CI}=0.49-3.56)$.

Conclusion: In our setting, head or spine injury significantly increases the hazard of mortality significantly compared to all other anatomic injury locations. The prioritization of timely operative and non-operative head injury management is imperative. The development of head injury units may help attenuate trauma- related mortality in resource poor settings.
\end{abstract}

\section{Introduction}

Injury is a significant cause of mortality worldwide, accounting for $10 \%$ of annual deaths globally, with millions more disabled [1,2]. In 2015, injuries caused 4.7 million deaths [2] and 247 million disability adjusted life years (DALYs) [1]. The three leading global causes of injury related deaths are road traffic crashes, suicide, and

\footnotetext{
* Corresponding author at: UNC School of Medicine, Department of Surgery, University of North Carolina, 4008 Burnett Womack building CB 7228 United States. E-mail address: anthchar@med.unc.edu (A. Charles).
}

homicide, which currently rank as the $9^{\text {th }}, 16^{\text {th }}$ and $22^{\text {nd }}$ global leading causes of death, respectively. All are predicted to rise in rank compared to other causes of death, placing all three of these among the top 20 leading causes of death in the world by 2030 [3]. An estimated $90 \%$ of trauma-related deaths and DALYs occur in low and middle income countries (LMIC) [4].

Trauma in sub-Saharan Africa (SSA) is often a result of road traffic crashes, assaults, or falls. Primary prevention of these types of injuries is an important aspect of the public health approach to decreasing morbidity and mortality [5]. Secondary prevention of injury-related morbidity and mortality should focus on 
strategically optimizing care of these patients. In a setting with limited resources, we must understand the most significant drivers of mortality in order to prioritize targeted interventions. Morbidity and mortality associated with trauma is driven in part by injury mechanism, and in part by the anatomic location of injuries [6].

Management priorities and risk of mortality vary greatly based on the anatomic location of injury. Clearly, injuries that affect airway and breathing, including head and neck injuries, as well as thoracic trauma, should take priority, as their impact on survival is more time-sensitive [7]. These injuries should therefore be given first priority during initial resuscitation. Because of the critical nature of these injuries, both head and chest injuries have been shown to be associated with significant mortality. Injury to the central nervous system is estimated to be the most common cause of trauma-related death [8], responsible for one-third to one-half of all trauma related mortality [9], while reports of thoracic trauma in SSA estimate that up to $15 \%$ of patients with blunt injury to the chest die before or within an hour of reaching the hospital [10]. Abdominal trauma in this setting also represents a significant cause of mortality, presenting a risk of internal hemorrhage or hollow viscous injury [11] that can be difficult to address with limited operative resources and blood banking.

Due to the significance of injury as a global public health concern, we aimed to characterize trauma mortality, and ascertain the hazard of mortality associated with anatomic location of injury in our setting at Kamuzu Central Hospital, Malawi. We believe that this will help inform trauma care in a resource-poor setting. Based on existing literature on the most common causes of death following trauma [8,9], we hypothesized that injury to the central nervous system would confer an increased risk for mortality, after adjusting for significant covariates, when compared to injuries in other anatomical locations.

\section{Materials and methods}

We performed a retrospective analysis of a prospectively collected dataset utilizing the trauma registry at Kamuzu Central Hospital, in Lilongwe, Malawi from 2011 to 2015. We included admitted adults ( $\geq 16$ years), and excluded patients who were treated as outpatients or were brought in dead. Children were excluded in this analysis as the pattern of trauma tends to be different in the pediatric population. Characteristics of the $\mathrm{KCH}$ trauma surveillance registry have been previously described [12].

\section{Setting}

$\mathrm{KCH}$ is a 1000 -bed tertiary care center with a catchment area of 6 million people in Lilongwe, Malawi. Ultrasounds, x-rays, and computerized tomography (CT) scans, as well as limited laboratory investigations and a blood bank, are available. Both radiology and laboratory assets are subject to personnel, electricity and supply availability, and are therefore not always fully functional.

Teams of interns, general surgery residents, and consultant general surgeons manage most trauma patients at $\mathrm{KCH}$. One neurosurgeon, one pediatric surgeon, and one urologist are on staff. Several consultant orthopedic surgeons work with a team of clinical officers to manage orthopedic injuries. Clinical officers are licensed medical practitioners with a relatively shortened training time that make up a large proportion of the healthcare workforce in Malawi [13]. All anesthesiologists are also clinical officers. $\mathrm{KCH}$ has a high-dependency, or step-down, unit equipped with electronic monitoring, and a lower nurse to patient ratio, as well as a five-bed intensive care unit with ventilator capability, staffed by nurses and clinical officers.

Up to three injuries per patient are recorded in the database, described by type (for example, contusion, laceration, fracture, etc), and by anatomic location. The patient's most severe injury, as judged by the evaluating clinician, is designated as Injury 1, followed by the second- and third-most severe injuries, if applicable. We stratified the patients based on the anatomic location of Injury 1, into head/spine, chest, abdomen, upper extremity, or lower extremity/pelvis. Head/spine patients had their most severe injury to the head, neck, back, spine, or face.

We performed descriptive statistics based on the anatomic location of the patient's most severe injury. Statistical significance of differences between groups was assessed using Pearson's chisquare test for categorical variables, and ANOVA for continuous variables. We performed bivariate analysis based on mortality to identify covariates that were significantly associated with increased mortality. We then constructed a Cox proportional hazards model. We selected upper extremity injury as the reference group, expecting these injuries to confer the lowest hazard of mortality. We adjusted the model for age, sex, injury severity, transfer status, injury mechanism, and surgical intervention based on variables that were significant on bivariate analysis. Injury severity was established using the Revised Trauma Score (RTS), which includes the Glasgow Coma Score (GCS), systolic blood pressure, and respiratory rate. Adjusted hazard ratios were used to calculate the

Table 1

Characteristics of Patients and Trauma by Anatomic Injury Location.

\begin{tabular}{|c|c|c|c|c|c|}
\hline & Head or Spine & Chest & Abdomen & Upper Extremity & Lower Extremity/Pelvis \\
\hline Number of patients $($ Total $=8569$ ) & $(3712[43.6 \%])$ & $(403[4.7 \%])$ & $(507[6.0 \%])$ & $(1329[15.6 \%])$ & $(2564[30.1 \%])$ \\
\hline Age (8530 [99.5\%]) (years) & 33.5 & 36.1 & 31.3 & 34.4 & 40.5 \\
\hline Male Sex (8564 [99.9\%]) & $3152(85.0 \%)$ & $333(82.6 \%)$ & $415(81.9 \%)$ & $1053(79.2 \%)$ & $1891(73.8 \%)$ \\
\hline \multicolumn{6}{|l|}{ Setting of Injury (8507 [99.3\%]) } \\
\hline Home & $723(19.6 \%)$ & $104(26.3 \%)$ & $126(25.1 \%)$ & $358(27.1 \%)$ & $765(30.0 \%)$ \\
\hline Work & $201(5.5 \%)$ & $22(6.3 \%)$ & $33(6.6 \%)$ & $134(10.2 \%)$ & $163(6.4 \%)$ \\
\hline Road/Street & $2380(64.6 \%)$ & $223(56.3 \%)$ & $249(49.6 \%)$ & $657(49.8 \%)$ & $1281(50.3 \%)$ \\
\hline Public Space & $231(6.3 \%)$ & $25(6.3 \%)$ & $51(10.2 \%)$ & $75(5.7 \%)$ & $97(3.8 \%)$ \\
\hline Other & $231(6.3 \%)$ & $22(6.3 \%)$ & $43(8.6 \%)$ & $95(7.3 \%)$ & $243(9.5 \%)$ \\
\hline \multicolumn{6}{|l|}{ Mechanism of Injury (8527 [99.7\%]) } \\
\hline Pedestrian in RTA & $484(13.1 \%)$ & $36(9.0 \%)$ & $41(8.1 \%)$ & $88(6.6 \%)$ & $368(14.5 \%)$ \\
\hline Driver/passenger in RTA & $1230(33.3 \%)$ & $134(33.5 \%)$ & $128(25.3 \%)$ & $387(14.8 \%)$ & 729 (28.6\%) \\
\hline Fall & $230(6.2 \%)$ & $26(6.5 \%)$ & $39(7.7 \%)$ & $206(15.6 \%)$ & $736(28.9 \%)$ \\
\hline Assault & $1429(38.7 \%)$ & $143(35.8 \%)$ & $212(41.9 \%)$ & $365(27.6 \%)$ & $306(12.0 \%)$ \\
\hline Other & $323(8.7 \%)$ & $61(15.3 \%)$ & $86(17.0 \%)$ & $279(21.1 \%)$ & 407 (16.0\%) \\
\hline Presented within $4 \mathrm{~h}$ of injury (6973 [81.4\%]) & $1774(58.1 \%)$ & $206(60.1 \%)$ & $194(45.9 \%)$ & $520(47.3 \%)$ & $852(41.5 \%)$ \\
\hline Alcohol Involved? (8549 [99.8\%]) & $443(12.0 \%)$ & $42(10.5 \%)$ & $67(13.2 \%)$ & $97(11.9 \%)$ & $164(6.4 \%)$ \\
\hline Transfer from outside hospital? (8490 [99.1\%]) & $1395(37.7 \%)$ & $152(37.8 \%)$ & $252(49.8 \%)$ & $546(41.2 \%)$ & $1183(46.3 \%)$ \\
\hline
\end{tabular}


mortality probability of head and spine injury patients. Finally, we constructed a survival analysis curve to establish differences in survival over time, based on anatomic location of injury. Our survival analysis spanned from the time of the injury to in hospital death or discharge. Sensitivity analysis was performed for missing data in the patient cohort between survivors and non-survivors particular for GCS and RTS. Data was missing at random in both groups. Descriptive statistics were used to describe the trend in incidence and mortality of head and spine injuries over five years. All analyses were performed using Stata/IC 14.1 (StataCorp, College Station, TX). Ethics approval was obtained from the University of North Carolina Institutional Review Board and the Malawi National Health Services Review Committee.

\section{Results}

From 2011-2015, a total of 76,984 trauma patients presented to $\mathrm{KCH}$ (Table 1). Of these, 49,126 (63.8\%) were adults and 8569 (17.4\%) were admitted. The most common injury was to the head or spine, seen in 3712 patients (43.6\%), followed by injury to the lower extremity/pelvis in 2564 (30.1\%), the upper extremity in 1329 (15.6\%), abdomen in 507 (6.0\%), and chest in 403 (4.7\%). Mean patient age was $35.7 \pm 14.9$ years. There was a male preponderance in the population, at 6872 (80.2\%), and male patients far exceeded female in all injury locations.

Head/spine injuries were least likely to occur at home, and most likely to have occurred on the road. While the road was the most common injury setting in all groups, other groups had a more even distribution among the home, work, and public space settings. Road traffic crashes were the most common cause of injury in all groups, except those with abdominal or upper extremity injuries. In these groups, the most common etiology was assault. Patients with extremity trauma had more falls as the etiology of the traumatic injury. Head, spine, and chest injury patients were more likely than other patients to have presented within four hours of injury occurrence, with lower extremity/pelvis trauma patients being most likely to present outside of this window. Alcohol involvement was highest in abdominal trauma. Abdomen and lower extremity/pelvis patients were most likely to have presented to a district hospital and then be transferred to $\mathrm{KCH}$, while head and spine patients were most likely to have come to $\mathrm{KCH}$ from the scene of injury.
The RTS [14] was calculated to assess overall injury severity (Table 2). According to the RTS, patients with primary abdominal injury had the least severe injuries at presentation, followed by head/spine patients. Patients with extremity trauma were the most likely to have a low RTS (3-10), indicative of a more severe injury, with $26.0 \%$ of lower extremity and $27.1 \%$ of upper extremity injuries scoring in this range. All other groups had less than $22 \%$ of patients scoring a low RTS.

Predictably, head and spine injury patients tended to have lower GCS than patients with injuries to other anatomic locations (Table 2). Head and spine injury patients were mostly likely to require care in the intensive care unit and high dependency units, at rates of $3.6 \%$ and $5.9 \%$, respectively. Patients with abdominal trauma were more likely to be managed operatively than patients with chest or head trauma.

Trauma mortality in the casualty department was $1.8 \%$ (Table 2 ). Casualty department mortality was highest in the head/spine patients, at $2.8 \%$, followed by abdominal patients at $2.4 \%$, thoracic patients at $2.2 \%$, upper extremity patients at $1.0 \%$, and lower extremity/pelvis patients at $0.6 \%$. Overall mortality for all admitted adult trauma patients was $6.0 \%$, with head/spine injuries again causing the highest mortality at $9.2 \%$, followed by abdominal at $7.5 \%$, thoracic at $4.2 \%$, upper extremity at $3.3 \%$, and lower extremity/pelvis patients at $2.8 \%$ (Fig. 1).

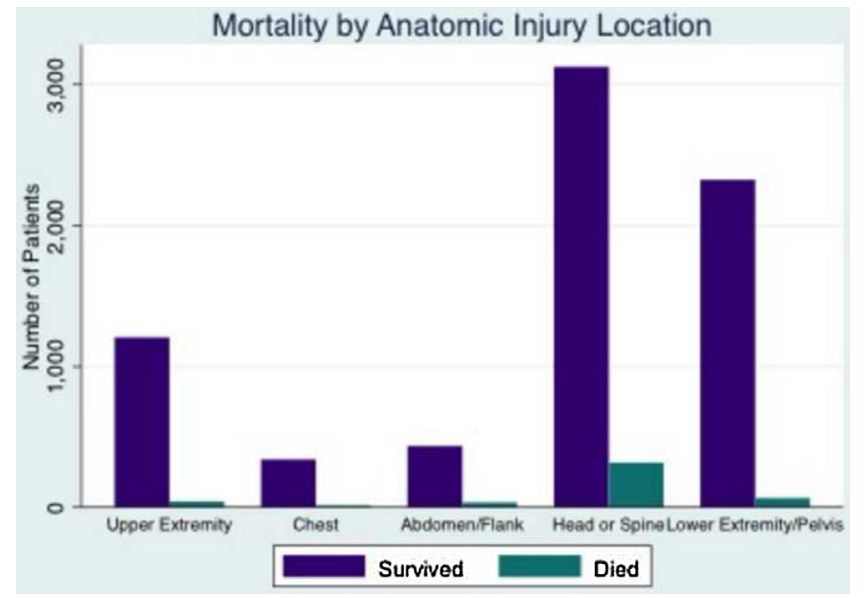

Fig. 1. Total patient mortality by anatomic location of most severe injury.

Table 2

Management and Outcomes by Anatomic Injury Location.

\begin{tabular}{|c|c|c|c|c|c|}
\hline & Head or Spine & Chest & Abdomen & Upper Extremity & Lower Extremity/Pelvis \\
\hline \multicolumn{6}{|l|}{ Revised Trauma Score (6161 [71.9\%]) } \\
\hline 12 (delayed care) & $1974(73.7 \%)$ & $200(72.2 \%)$ & $266(75.1 \%)$ & $701(70.5 \%)$ & $1298(71.4 \%)$ \\
\hline 11 (immediate care) & $136(5.1 \%)$ & $17(6.1 \%)$ & $17(4.8 \%)$ & $24(2.4 \%)$ & $48(2.6 \%)$ \\
\hline 3-10 (urgent care) & $569(21.2 \%)$ & $60(21.7 \%)$ & $71(20.1 \%)$ & $270(27.1 \%)$ & $473(26.0 \%)$ \\
\hline \multicolumn{6}{|l|}{ Glasgow Coma Score (6664 [77.8\%]) } \\
\hline $14-15$ & $2725(92.0 \%)$ & 322 (99.1\%) & $384(97.5 \%)$ & $1003(98.1 \%)$ & 1897 (98.9\%) \\
\hline $9-13$ & $105(3.6 \%)$ & $2(0.6 \%)$ & $8(2.0 \%)$ & $11(1.1 \%)$ & $11(0.6 \%)$ \\
\hline$\leq 8$ & $131(4.4 \%)$ & $1(0.3 \%)$ & $2(0.5 \%)$ & $8(0.8 \%)$ & $11(0.6 \%)$ \\
\hline \multicolumn{6}{|l|}{ Admission Disposition (8487 [99.0\%]) } \\
\hline Admitted & $3599(97.2 \%)$ & $394(97.8 \%)$ & $490(97.6 \%)$ & $1310(99.0 \%)$ & $2544(99.5 \%)$ \\
\hline Died in Casualty & $102(2.8 \%)$ & $9(2.2 \%)$ & $12(2.4 \%)$ & $13(1.0 \%)$ & $14(0.6 \%)$ \\
\hline \multicolumn{6}{|l|}{ Highest Level of Care } \\
\hline Ward & $3203(90.5 \%)$ & $376(96.4 \%)$ & $452(93.6 \%)$ & $1263(98.7 \%)$ & $2439(98.7 \%)$ \\
\hline HDU & $210(5.9 \%)$ & $8(2.1 \%)$ & $18(3.7 \%)$ & $11(0.9 \%)$ & $13(0.5 \%)$ \\
\hline ICU & $126(3.6 \%)$ & $6(1.5 \%)$ & $13(2.7 \%)$ & $6(1.5 \%)$ & $20(0.8 \%)$ \\
\hline Underwent Surgery (6694 [78.1\%]) & 1115 (38.9\%) & $108(36.7 \%)$ & $220(57.9 \%)$ & $398(36.1 \%)$ & $599(29.6 \%)$ \\
\hline \multicolumn{6}{|l|}{ Final Outcome (7949 $[92.8 \%])$} \\
\hline Death & $317(9.2 \%)$ & $15(4.2 \%)$ & $35(7.5 \%)$ & $41(3.3 \%)$ & $66(2.8 \%)$ \\
\hline Discharge & $2910(84.5 \%)$ & $321(90.4 \%)$ & 407 (86.6\%) & 1139 (91.3\%) & $2229(93.3 \%)$ \\
\hline Lost/Transferred/Absconded & $216(6.3 \%)$ & $19(5.4 \%)$ & $28(6.0 \%)$ & $67(5.4 \%)$ & $95(4.0 \%)$ \\
\hline
\end{tabular}


Table 3

Bivariate Analysis by Mortality of Total Population.

\begin{tabular}{|c|c|c|c|}
\hline & Survived $(\mathrm{n}=7473[94.0 \%])$ & $\operatorname{Died}(n=476[6.0 \%])$ & p-Value \\
\hline Age & $35.6 \pm \underline{15.0}$ & $38.9 \pm \underline{15.4}$ & 0.000 \\
\hline Male Sex & $6019(\overline{80.6} \%)$ & $393(8 \overline{2.9 \%})$ & 0.207 \\
\hline Injury Setting & & & 0.000 \\
\hline Home & $1843(24.8 \%)$ & $94(20.1 \%)$ & \\
\hline Work & $487(6.6 \%)$ & $19(4.1 \%)$ & \\
\hline Road/Street & $4140(55.7 \%)$ & $311(66.5 \%)$ & \\
\hline Public Space & $436(5.9 \%)$ & $21(4.5 \%)$ & \\
\hline Other & $528(7.1 \%)$ & $23(4.9 \%)$ & \\
\hline Blunt Mechanism of Injury & $7046(94.7 \%)$ & $459(96.6 \%)$ & 0.068 \\
\hline Injury Season & & & 0.144 \\
\hline Rainy & $1702(22.8 \%)$ & $90(18.9 \%)$ & \\
\hline Lush, green & $1827(24.5 \%)$ & $111(23.3 \%)$ & \\
\hline Cold, dry & $1061(27.8 \%)$ & $141(29.6 \%)$ & \\
\hline Hot, dry & $1883(93.4 \%)$ & $134(6.6 \%)$ & \\
\hline Time to Presentation & & & 0.000 \\
\hline $0-4 \mathrm{~h}$ & $3046(50.2 \%)$ & $209(56.8 \%)$ & \\
\hline $5-8 h$ & $635(10.5 \%)$ & $49(13.3 \%)$ & \\
\hline $8-16 \mathrm{~h}$ & $521(8.6 \%)$ & $38(10.3 \%)$ & \\
\hline $17-24 \mathrm{~h}$ & $435(7.2 \%)$ & $24(6.5 \%)$ & \\
\hline $25-48 \mathrm{~h}$ & $511(8.4 \%)$ & $19(5.2 \%)$ & \\
\hline $49-96 h$ & $366(6.0 \%)$ & $13(3.5 \%)$ & \\
\hline$>96 \mathrm{~h}$ & $553(9.1 \%)$ & $16(4.4 \%)$ & \\
\hline Alcohol Use & $698(9.4 \%)$ & $45(9.5 \%)$ & 0.919 \\
\hline Transfer Status & $3110(41.7 \%)$ & $222(46.6 \%)$ & 0.035 \\
\hline RTS & & & 0.000 \\
\hline 12 & $3382(60.0 \%)$ & $126(36.0 \%)$ & \\
\hline 11 & $210(3.7 \%)$ & $23(6.6 \%)$ & \\
\hline $3-10$ & $2046(36.3 \%)$ & $201(57.4 \%)$ & \\
\hline Glasgow Coma Score & & & 0.000 \\
\hline $14-15$ & $5790(97.3 \%)$ & $226(68.9 \%)$ & \\
\hline $9-13$ & $92(1.6 \%)$ & $26(7.9 \%)$ & \\
\hline$\geq 8$ & $66(1.1 \%)$ & $76(23.2 \%)$ & \\
\hline Highest Level of Care & & & 0.000 \\
\hline Ward & $7112(96.8 \%)$ & $158(49.4 \%)$ & \\
\hline HDU & $168(2.3 \%)$ & $78(24.4 \%)$ & \\
\hline ICU & $68(0.9 \%)$ & $84(26.3 \%)$ & \\
\hline Underwent Surgical Intervention & $2333(31.2 \%)$ & $100(21.0 \%)$ & 0.000 \\
\hline Anatomic Injury Location & & & 0.000 \\
\hline Head/Spine & $3126(42.1 \%)$ & 317 (66.9\%) & \\
\hline Chest & $340(4.6 \%)$ & $15(3.2 \%)$ & \\
\hline Abdomen & $435(5.9 \%)$ & $35(7.4 \%)$ & \\
\hline Upper Extremity & $1206(16.2 \%)$ & $41(8.7 \%)$ & \\
\hline Lower Extremity/Pelvis & $2324(31.3 \%)$ & $66(13.9 \%)$ & \\
\hline
\end{tabular}

On bivariate analysis (Table 3), patient age, injury setting, time to presentation, transfer status, RTS, GCS, highest level of care, and surgical intervention were statistically significantly different between survivors and non-survivors. Anatomic location of injury also varied significantly between the two groups. Head/spine patients comprised the largest proportion of the deceased population at $67 \%$, followed by lower extremity patients at $13.9 \%$, upper extremity patients at $8.7 \%$, abdominal patients at 7.4\%, and chest patients at 3.2\% (Table 3 ).

A Cox proportional hazards model using upper extremity as the baseline comparator demonstrated the highest unadjusted and adjusted hazard ratios of mortality in head/spine injury patients. Unadjusted $\mathrm{HR}$ was $\mathrm{HR}=3.685(95 \% \mathrm{CI}=2.50-5.44)$, which rose to $4.501(95 \% \mathrm{CI}=2.78-7.30)$ when adjusted for age, sex, RTS, transfer status, injury mechanism, and surgical intervention. In the adjusted model, abdominal trauma carried the second highest hazard of mortality at $3.621(95 \% \mathrm{CI}=1.92-6.84)$ followed by thoracic trauma ( $\mathrm{HR}=1.362195 \% \mathrm{CI}=0.49-3.56)$ and lower extremity/pelvis trauma $(\mathrm{HR}=0.4186,95 \% \mathrm{CI}=0.23-0.76$ ).

Using the hazard ratio determined by the fully adjusted model, a probability of mortality for each injury was calculated (Table 4). The associated probability of mortality for head/spine injury was $81.8 \%$. In survival analysis (Fig. 2), abdominal trauma had the highest effect on mortality during the days immediately following the injury, but head and spine injuries had a much more pronounced mortality over during the subsequent weeks and months. Data across five years (Figs. 3 and 4) showed that while incidence of head/spine trauma decreased at a rate of $5.2 \%$ per year,

Table 4

Hazard of Mortality by Anatomic Injury Location.

\begin{tabular}{|c|c|c|c|c|}
\hline & Hazard Ratio & Associated Probability of Mortality & P-Value & Confidence Interval \\
\hline Head or Spine & 4.50 & $81.8 \%$ & 0.000 & $2.77-7.29$ \\
\hline Chest & 1.33 & $57.0 \%$ & 0.575 & $0.49-3.56$ \\
\hline Abdomen & 3.62 & $78.4 \%$ & 0.000 & $1.91-6.84$ \\
\hline Upper Extremity & 1.00 & $50.0 \%$ & & \\
\hline Lower Extremity/Pelvis & 0.42 & $29.5 \%$ & 0.004 & $0.23-0.76$ \\
\hline
\end{tabular}




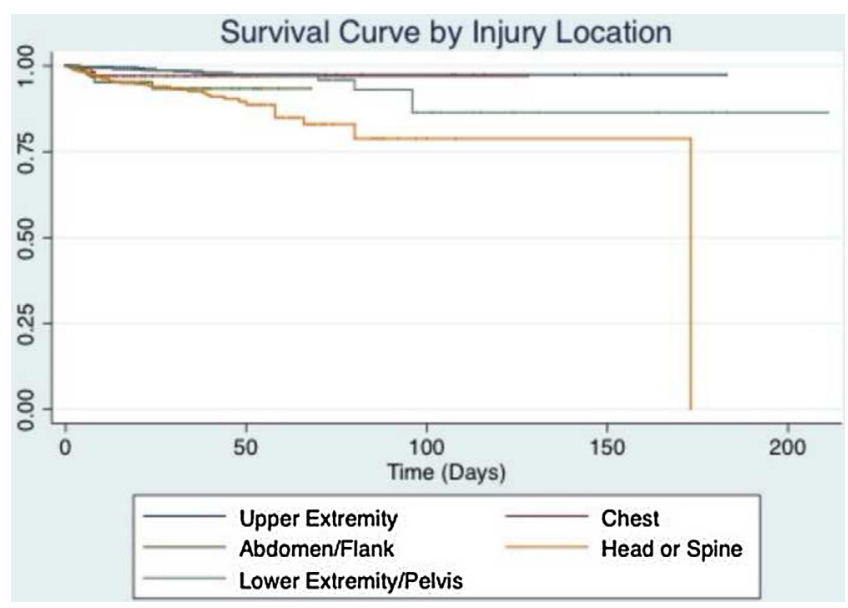

Fig. 2. Survival time curve by anatomic location of most severe injury. mortality in head/spine injury patients has steadily increased at a rate of $3.8 \%$ per year.

\section{Discussion}

In this study, we analyzed a well-established trauma registry in a low-resource setting. By stratifying our population into anatomic location of the most severe injury, and using the upper extremity as the reference, we were able to determine the hazard ratio of mortality for each anatomic location. To our knowledge, this is the first comparative analysis of its kind in a trauma cohort in SSA.

Our analysis revealed that the most severe injuries occurred in extremity patients, as shown by the relatively low RTS in these groups (Table 2). This may be explained by the tendency of severe extremity injuries to occur in the context of polytrauma. While these patients were stratified to the upper or lower extremity cohort based on their most severe injury, they may have had other less visible, but more severe injuries that may have complicated

Incidence Over Time by Anatomic Injury Location

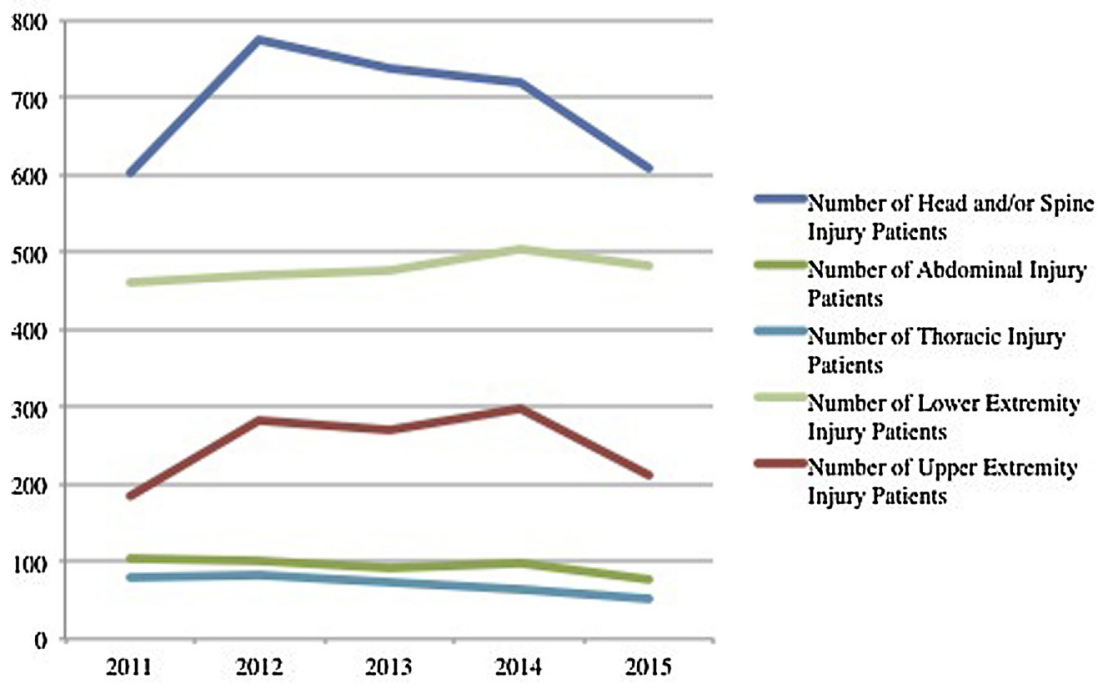

Fig. 3. Incidence of injury over time by anatomic location.

\section{Mortality Over Time by Anatomic Injury Location}

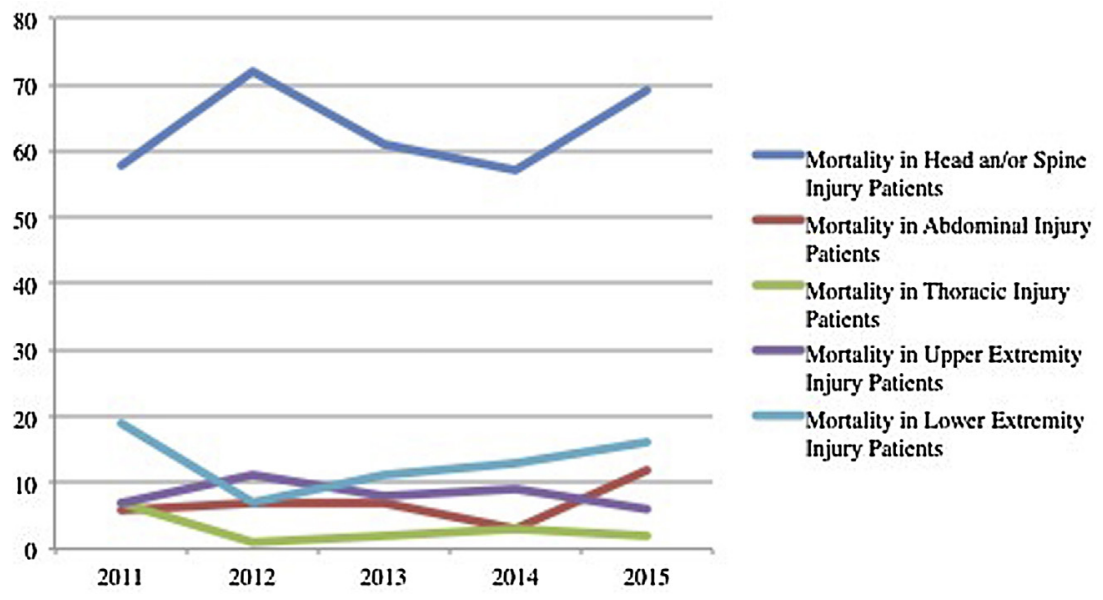

Fig. 4. Injury Mortality injury over time by anatomic location. 
their care. Despite this, these two groups of patients had the lowest mortality (Table 2).

Patients with abdominal trauma were most likely to have undergone surgery. This may be influenced by the fact that surgeons at $\mathrm{KCH}$ are best equipped to perform laparotomies, versus thoracotomies or craniotomies. Additionally, it can be seen in the survival analysis (Fig. 2) that abdominal patients suffer from the worst outcomes in the days immediately following the injury, but head/spine patients have much worse long-term survival rates. This is reflective of the debilitating long-term effects of central nervous system injury and the continuous care required by these patients. Even late in the patients' hospital stay, head/spine patients continued to have significant mortality, as shown by the late drop-offs in the survival curve. Overall, we found that the presence of head or spine injury confers a four-fold increase in the hazard of mortality compared to upper extremity trauma, followed by abdominal and thoracic injures.

Our study highlights the public health significance of traumatic brain injury (TBI) and spinal cord injury (SCI) in a resource poor setting, and confirms the findings of other researchers, who demonstrate that TBI is a leading cause of death and disability with more than 10 million people affected each year [15]. Injury to central nervous system-brain and spinal cord-remains the most common cause of death in trauma patients worldwide [8]. In a study by Sauaula et al. [16] that evaluated 289 post-injury fatalities, central nervous system injury was found to be the most frequent cause of death, comprising $42 \%$ of total mortality. Similarly, a retrospective review of autopsy reports in Norway reported central nervous system injury as the cause of death in $67 \%$ of trauma mortalities [17].

The literature on the prevalence of trauma in sub-Saharan Africa remains scarce, and the studies reported are relatively small. As such, the burden of central nervous system injury in this region is likely underestimated. Reports from South Africa [18], Kenya [19], Tanzania [20], and Uganda [21] each describe populations ranging from 90 to a few hundred patients. Because of our existing trauma database, we were able to assess a much larger population. In our population of 8569 trauma patients, we found that $66.9 \%$ of mortality was attributable to head/spine injury.

The global burden of TBI/SCI cannot be attenuated if this region is ignored. While the global average incidence is estimated at 200 per 100,000 people annually, rates of TBI/SCI vary according to region [22]. The only report on total population-based prevalence in SSA, from Johannesburg in 1991, estimated an annual TBI incidence of 316 per 100,000 [23]. One modeling study estimates that by 2050 , Africa alone will have a burden of 6-14 million new cases of TBI per year [24]. This is attributable to increasing motorized transportation, violence, and persistent armed conflicts in a region where access to neurosurgical care remains very limited [25].

Low- and middle-income countries (LMICs) suffer from the double burden of disproportionately high incidence of, and mortality from, TBI/SCI. One analysis found that patients in LMICs have more than twice the odds of mortality after severe head injuries compared with patients in high-income countries [26]. In addition to mortality, TBI and SCI are each associated with significant morbidity ranging from physical deficits to neurocognitive disabilities or epilepsy, with rates of moderate to severe disability among mild to severe TBI patients as high as $48 \%$ at one year [27]. Spinal cord trauma often produces permanent quadriparesis or paraparesis. Because the victims of TBI and SCI tend to be young men at the most productive time of their lives, the resultant trauma related morbidity and mortality has negative economic impact locally and nationally.

Optimal management of TBI at the first point of contact with the healthcare system entails prevention, or early correction, of secondary injury caused by cerebral edema from hypoxia, hypoglycemia, hyperthermia, infection, and hypotension. Evidence-based international guidelines for the best practices in managing these patients are available [28], however, they are not always feasible to implement in resource-poor settings. In the high income countries, patients managed in a non-neurosurgical center have more than twice the odds of death compared to those treated in a neurosurgical center [29], with a neurosurgical center being defined as a hospital with 24-h coverage by a neurosurgeon, and all necessary diagnostic and therapeutic adjuncts available. However, because there is only 1 neurosurgeon per $10,000,000$ people in Africa, as compared to 102 per $10,000,000$ in Europe and 56 per $10,000,000$ globally, [30] a neurosurgical center is not available to the majority of the SSA population.

Even in the presence of a low surgical workforce in resource poor countries, effective care of TBI patients is still achievable. Most TBI patients do not need surgical management, but require intensive monitoring and adherence to protocols. Recent studies from South Africa [31] and Kenya [21] have developed simple, lowcost TBI management protocols for resource-poor settings, which include standard recommendations such as a thorough history and exam, regular assessment of neurological function, and elevation of head of bed. However, studies from SSA centers that implemented these protocols have revealed low adherence. Optimum TBI management in low-resource setting thus requires education of clinicians as well as resource allocation. The establishment of head injury units, with full-time coverage by carefully trained clinical staff utilizing well-defined protocols can help reduce TBI- related morbidity and mortality.

The limitations of our study are those inherent to any study with retrospective methodology. Given the nature of our database, missing data has the potential to affect our analysis. Of our variables of interest, GCS and RTS were the commonly missing values, at a rate of $22.2 \%$ and $30.1 \%$ missing, respectively; however, upon sensitivity analysis the variables appear to be missing at random, and we believe that due to the size of our patient cohort, the conclusions are unaffected. We are also limited by the nature of the database to using head injury as a proxy for TBI, and spine injury as a proxy for SCI. Head injury is a general term used to describe any trauma to the head, but is most significant when it involves the brain. TBI is defined as head injury causing an alteration in consciousness, or head injury with radiographic evidence of injury to the brain. In this study, given our lack of radiographic data and data on the patients' neurological exam beyond admission GCS, we equated the anatomic location of injury in the head, with TBI. Likewise, SCI refers to damage to the spinal cord itself, as opposed to the bony spinal column or the surrounding soft tissues, which are included in the more general term spine injury. $\mathrm{SCI}$ is defined as temporary or permanent loss of motor, sensory, or autonomic function below the level of the lesion due to cord injury. However, given our method of classifying injuries only by anatomic location and without clinical or radiographic data, we were only able to consider the more general spine injury here. Additionally, in this study, we attribute cause of death to the anatomical region that is most severely injured. It is possible that death was caused by non-injury related factors.

\section{Conclusion}

Head or spine injury is common, and often results in significant mortality compared to injury to other anatomic locations. In our resource poor setting, the presence of head or spine injury significantly increases the hazard of mortality after controlling for significant covariates. The prioritization of a timely protocol-based management of head injured patients is imperative to attenuate global trauma-related mortality. This may best be implemented by 
establishing dedicated head injury units in hospitals in lowresource settings.

\section{Acknowledgements}

The authors report no conflicts of interest concerning the materials or methods used in this study or the findings specified in this paper. This work was supported by the Fulbright Program and the National Institutes of Health Office of the Director, Fogarty International Center, Office of AIDS Research, National Cancer Center, National Heart, Blood, and Lung Institute, and the NIH Office of Research for Women's Health through the Fogarty Global Health Fellows Program Consortium comprised of the University of North Carolina, John Hopkins, Morehouse and Tulane (R25TW009340).

\section{References}

[1] Murray CJ, Barber RM, Foreman KJ, Abbasoglu OA, Abd-Allah F, Abera SF, et al. Global, regional and national disability-adjusted life years (DALYs) for 306 diseases and injuries and healthy-life expectance for 188 countries, 19902013: quantifying the epidemiological transition. Lancet 2015;386.

[2] Wang HNM, Allen C, Barber RM, Bhutta ZA, Carter A, Casey DC, et al. Global, regional, and national life expectancy, all-cause mortality, and cause-specific mortality for 249 causes of death, 1980-2015: a systematic analysis for the Global Burden of Disease Study 2015. Lancet 2016;388:1459-544.

[3] Mathers C, Fat DM, Boerma JT, World Health Organization. The global burden of disease: 2004 update. World Health Organization; 2008.

[4] de Ramirez SS, Hyder AA, Herbert HK, et al. Unintentional injuries: magnitude, prevention, and control. Annu Rev Public Health 2012;33:175-91.

[5] Nordberg E. Injuries as a public health problem in sub-Saharan Africa: epidemiology and prospects for control. East Afr Med J 2000;77(12):S1-43.

[6] Fani-Salek MHTV, Terezakis SA. Trauma scoring systems explained. Emerg Med 1999;11:155-66.

[7] Tchorz KMAS. Advanced trauma life support (ATLS): the ninth edition. J Trauma Acute Care Surg 2013:74(5):1363.

[8] Capone-Neto A, Rizoli SB. Linking the chain of survival: trauma as a traditional role model for multisystem trauma and brain injury. Curr Opin Crit Care 2009;15(4):290-4.

[9] Daniel M, Sosin JES, Waxweiler Richard J. Trends in death associated with traumatic brain injury, 1979 through 1992: success and failure. JAMA 1995;273 (22):1778-80.

[10] Thomas MO, Ogunleye EO. Etiopathology and management challenges of blunt chest trauma in Nigeria. Asian Cardiovasc Thorac Ann 2009;17(6):608-11.

[11] Mnguni DM MN, Madiba TE. Adbominal trauma in durban, South Africa: factors influencing outcome. Int Surg 2012;97:161-8.

[12] Tyson AF, Varela C, Cairns BA, Charles AG. Hospital mortality following trauma: an analysis of a hospital-based injury surveillance registry in sub-Saharan Africa. J Surg Educ 2015;72(4):e66-72.
[13] van Amelsfoort vLP JJ, Jiskoot P, Ratsma YE. Surgery in Malawi-the training of clinical officers. Trop Doc 2010;40(2):74-6.

[14] Howard R, Champion WJS, Copes Wayne S, Gann Donald S, Gennarelli Thomas A, Flanagan Maureen E. A revision of the trauma score. Journal Trauma 1989;29 (5):623-9.

[15] Hyder AAWC, Puvanachandra P, Guruaj G, Kobusingye OC. The impact of traumatic brain injuries: a global perspective. NeuroRehabilitation 2007;22 (5):341-53.

[16] Sauala A, Moore Frederick A, Moore Ernest E, Moser Kathe S, Brennan Regina Read Robert A, Pons Peter T. Epidemiology of trauma deaths: a reassessment. J Trauma 1995;38(2):185-93.

[17] Soreide K, Kruger AJ, Vardal AL, Ellingsen CL, Søreide E, Lossius HM. Epidemiology and contemporary patterns of trauma deaths: changing place, similar pace, older face. World J Surg 2007;31(11):2092-103.

[18] Zulu BMW, Mulaudzi TV, Madiba TE, Muckart DJ. Outcome of head injuries in general surgical units with an off-site neurosurgical service. Injury 2007;38 (5):576-83.

[19] Mwita C, Muthoka J, Maina S, Mulingwa P, Gwer S. Early management of traumatic brain injury in a Tertiary hospital in Central Kenya: a clinical audit. J Neurosci Rural Pract 2016;7(1):97.

[20] Maier D, Njoku Jr. I, Schmutzhard E, Dharsee J, Doppler M, Härtl R, et al. Traumatic brain injury in a rural and an urban Tanzanian hospital-a comparative, retrospective analysis based on computed tomography. World Neurosurg 2014;81(3-4):478-82.

[21] Tran TM, Fuller AT, Kiryabwire J, Mukasa J, Muhumuza M, Ssenyojo H, et al. Distribution and characteristics of severe traumatic brain injury at Mulago National Referral Hospital in Uganda. World Neurosurg 2015;83(3):269-77.

[22] Roozenbeek B, Maas AI, Menon DK. Changing patterns in the epidemiology of traumatic brain injury. Nat Rev Neurol 2013;9(4):231-6.

[23] Nell V, Brown Digby SO. Epidemiology of traumatic brain injury in Johannesburg-II. Morbidity, mortality and etiology. Soc Sci Med 1991;33 (3):289-96.

[24] Wong JC, Linn KA, Shinohara RT, Mateen FJ. Traumatic brain injury in Africa in 2050: a modeling study. Eur J Neurol 2016;23(2):382-6.

[25] Maas AIR, Stocchetti N, Bullock R. Moderate and severe traumatic brain injury in adults. Lancet Neuro 2008;7(8):728-41.

[26] De Silva MJ, Roberts I, Perel P, Edwards P, Kenward MG, Fernandes J, et al. Patient outcome after traumatic brain injury in high-, middle- and low-income countries: analysis of data on 8927 patients in 46 countries. Int J Epidemiol 2009;38(2):452-8.

[27] Thornhill S. Disability in young people and adults one year after head injury: prospective cohort study. BMJ 2000;320:1631-5.

[28] Brain trauma foundation AAoNS, congress of neurological surgeons, AANS/CNS joint section of neurotrauma and critical care. guidelines for the management of severe traumatic brain injury. J Neurotrauma 2007;24(S1):1-106.

[29] Patel HC, Bouamra O, Woodford M, King AT, Yates DW, Lecky FE, et al. Trends in head injury outcome from 1989 to 2003 and the effect of neurosurgical care: an observational study. Lancet 2005;366(9496):1538-44.

[30] Janca A, Aarli JA, Prilipko L, Dua T, Saxena S, Saraceno B. WHO/WFN Survey of neurological services: a worldwide perspective. J Neurol Sci 2006;247(1):159.

[31] Alexander T, Fuller G, Hargovan P, Clarke DL, Muckart DJ, Thomson SR. An audit of the quality of care of traumatic brain injury at a busy regional hospital in South Africa. S Afr J Surg 2009;47(4):124-6. 